\title{
Design of Aquatic Ecological Security Evaluation System Based on Virtual Geographic Environment
}

\author{
Zengzheng Wang ${ }^{1, a}$, Chaoyang Fang ${ }^{1, b}$, Yeqiao Wang ${ }^{1, c}$ \\ ${ }^{1}$ College of Geography and Environment, Jiangxi Normal University, Nanchang, Jiangxi, China \\ a371228137@qq.com, b1036213442@qq.com, cywang0559@126.com
}

Keywords: Virtual geographic environment (VGE); Aquatic ecological security; Model Integration; Remote sensing image; Interactive Data Language(IDL)

Abstract. By building remote sensing for monitoring and evaluating water ecological security virtual geographical environment, in general it shows the system framework, integration and coordination models and visualization. It aims to explore for water ecological security and remote monitoring to assess the feasibility VGE key technologies to build. The results show that virtual geographic environment on the reproduction of complex geographic systems, forecasting and cognitive binding IDL powerful data visualization capabilities are an ideal tool for visualization of remote sensing images aquatic ecosystems and aquatic ecosystems safety monitoring and evaluation.

\section{Introduction}

Virtual geographical environment(VGE) refers to the digital real geographical environment, the historical environment of reproduction and restoration, and the future geographical environment of prediction and prediction. As a VGE of technology system, users can perceive dynamic geo-phenomena by means of multidimensional sensing and multi-channel feedback, and carry out experiments and explorations through geography modeling and simulation, geographic coordination, etc., to realize the complex geography system Reproduction, prediction and cognition.

In this paper, the water ecological security of Poyang Lake as the background, the use of VGE of Poyang Lake water ecological security simulation, to explore how the Poyang Lake region for remote sensing image water ecological security model integrated into the VGE. Analyzes the objectives, functions and realization of data environment, model environment, expression environment and collaborative environment. In the face of multidimensional dynamic geographical environment, a work platform for analyzing and understanding the geographical environment and monitoring water ecological security is designed and realized. It supports geometric, physical and modeling process requirements, and geo-visualization of model calculation results.

\section{System design}

System Objectives. Based on MODIS remote sensing data, such as high spectral resolution and high resolution, and a series of remote sensing image data of domestic high score 1, the model of water information extraction is adopted, and the principles of system robustness and extensibility are followed. using the IDL visual interactive language with image processing advantage, and constructing the water ecological security emergency monitoring system and related statistical visualization output information based on the VGE framework.

Overall system function framework. The system uses the more popular hierarchical structure, including the data layer, data processing layer, model layer and visualization layer. The core hardware architecture of the system is shown in Fig 1

Data environment. Data environment is mainly responsible for the organization and management of multi-source heterogeneous data, integration and integration, for the next model operation, visualization and geological analysis to provide data support. In this system, the data environment mainly corresponds to the data layer and the model layer in the hierarchical design of the system. 
The data layer mainly includes vector data, DEM data and remote sensing image data. The data processing layer is mainly used for the processing of raw data, including preprocessing of remote sensing data, pre-analysis of data and data format conversion, mainly adapting the original data to system operation, analysis, simulation and visualization.

All the modules in the system are database-centric, and the basic data such as remote sensing image, DEM data, vector data are stored in the database with spatial data read and stored, and read and written by database.

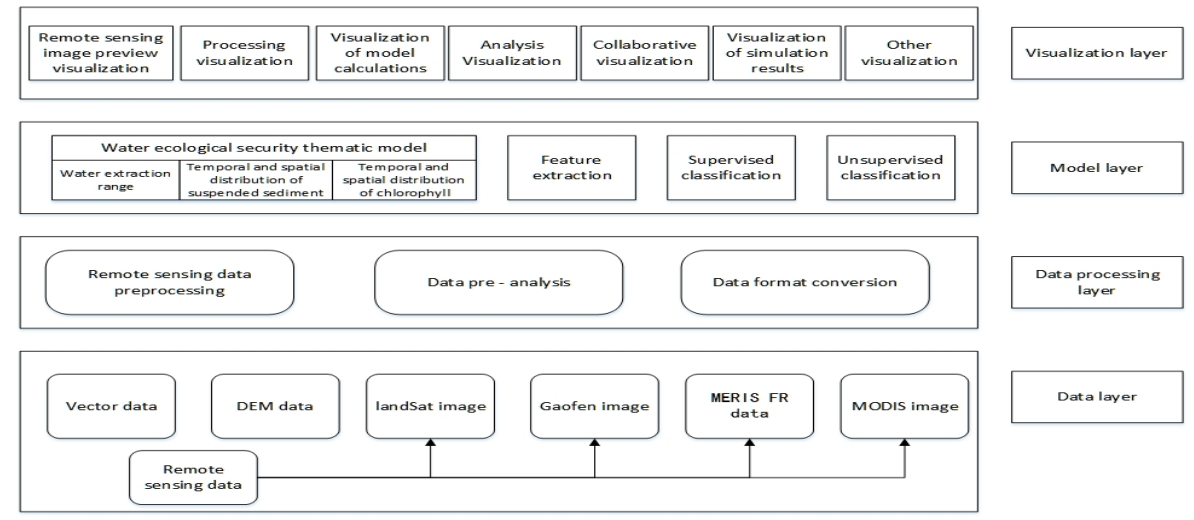

Fig 1 System overall framework diagram

Model environment. The model environment mainly corresponds to the model layer, which relates to multiple geospatial models, such as multiple circles of geographical environment, water body component extraction and so on. It is very important to analyze, simulate and forecast the geographical environment, and to construct and use the geographic model. The model layer mainly contains the various models needed in this study, and analyzes and classifies them. Second, in the system, the results of the model calculation need to be displayed quickly, the results of the model calculation simulation are placed in the model results database.

Performance environment. The performance environment mainly corresponds to visualization layer, which mainly includes remote visual image preview visualization, processing visualization, visualization of model calculation, analysis visualization, collaborative visualization, simulation result visualization and other visualization. It includes three-dimensional and two-dimensional visual linkage, visual expression of geographic data, multi-channel human-computer interaction based on visual, auditory, tactile and so on.

Collaborative environment. Collaborative environment mainly refers to the human-computer interaction, database and the collaboration between the model library and serves the multi-user communication, interaction and collaborative work. Database and model library through the background database management between the two to achieve synergy. Human-computer interaction and multi-user interaction between the collaboration based on the Virtual Geographic Environment simulation and decision support experimental area. Based on data, model-based, workflow-based and other geo-synergies, this paper builds a synergetic negotiation platform, aggregates knowledge representation and analysis synthesis, and provides decision support.

\section{Model integration}

The goal of virtual geospatial research is not to create specialized models. The goal is to effectively integrate the knowledge of these models with general knowledge and the new knowledge of the relevant regions. Model integration is the key to realize the VGE.

This paper constructs an integrated environment of water ecological security model based on satellite remote sensing data, and serves as a model server for VGE to provide high performance calculation for the model. In a VGE visualization platform. Use IDL language integrated operator terminal model on the computer, so that the complex model calculations and visualization platform separately, there is conducive to the management and maintenance of the system, provides easy 
high-performance computing and an operation and can be recycled platform for processing and computational models.

A three-layer model is designed, which is model server, intermediate management layer and user layer. Model calculations run on the model server. Users in the virtual geographical environment simulation and decision-making experimental area to operate and consult, responsible for generating the model operation instructions. Management is located between the experimental area and the server, responsible for the user's information conversion, filtering and shielding, thereby enhancing the security of the entire platform. Experimental area instruction issued after approval by management to convert into a model server command sent to the server model. The model server uses the parallel method to carry on the high performance fast computation to the model. The results are returned to the user through the interim management layer. Three- layer model of the layout structure shown in Fig 2.
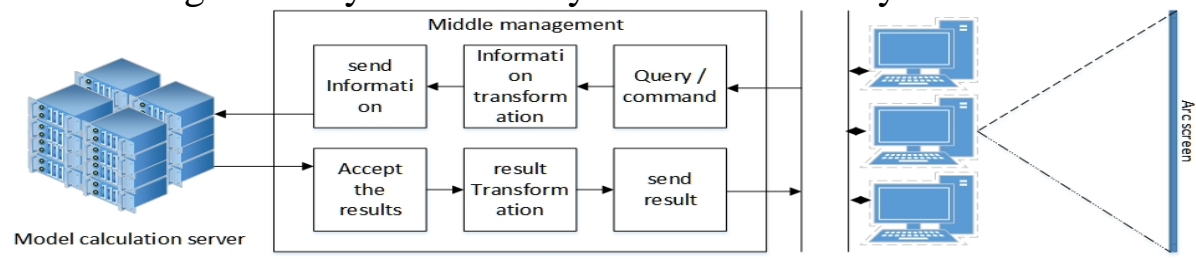

Fig 2 Three - layer model layout diagram

\section{Visualized Expression of Aquatic Ecological Security Assessment}

Water ecology security based on satellite remote sensing data should have good visual expression. Driven by the physical model, according to the law of change, this paper through 2D and 3D to express all kinds of aquatic ecological security model calculation and processing results.

On the basis of the basic realization of RS data visualization capabilities, combined with common GIS functions (zoom in / out, pan, Hawkeye, etc.), an increase of superimposed layers, basic drawing functions. The 2D platform is mainly for the integration of geographic information and water ecological security model processing results. The 3D environment can express all the $2 \mathrm{D}$ functions, and introduce the DEM data to express the processing results in 3D visualization, mainly using is surface construction. The platform adopts IDL language to realize the secondary development.

VGE Simulation and Decision Support Experiment area. The main equipment for the experimental area server room group, the arc screen projection, floor projection, the image of the wall, in consultation with the operating platform. The platform has two sets of giant screen projection screens, which are based on visual, auditory and tactile man-machine interaction, to enhance the immersive experience, integrate the Internet and mobile network, to carry out geographical experiment and avatar virtual collaborative seminar room. Which can be related to the field location experiment, indoor physical model experiment, correlation and complementation. It is also possible to construct a VGE based on certain geographic features, laws and phenomena. But also can be used as remote sensing data and product integration and shared services, visualization platform. You can also provide a three-dimensional digital model of the Earth's surface environment for remote sensing experiments, remote sensing data, as information on Remote Sensing Remote sensing mechanism modeling and simulation platform.

The system interface runs in the operation of the consultation platform, the data stored in the server room, the visual output of the results in the arc screen. 2D visualization can be projected with the 3D results in the platform, making it easy to find the connection between the different dimensions. The visual output of the 3D visualization is directly above the arc screen. Using the advantages of the large screen and high resolution, the result is more intuitive and convenient for more detailed observation of remote sensing images and model processing results and 3D visualization output results. At the same time, it can also play the role of multi-user coordination by using VGE simulation and decision support. The experts and researchers of different types can discuss together and facilitate decision-making. Experiment area configuration as shown in Fig 3. 


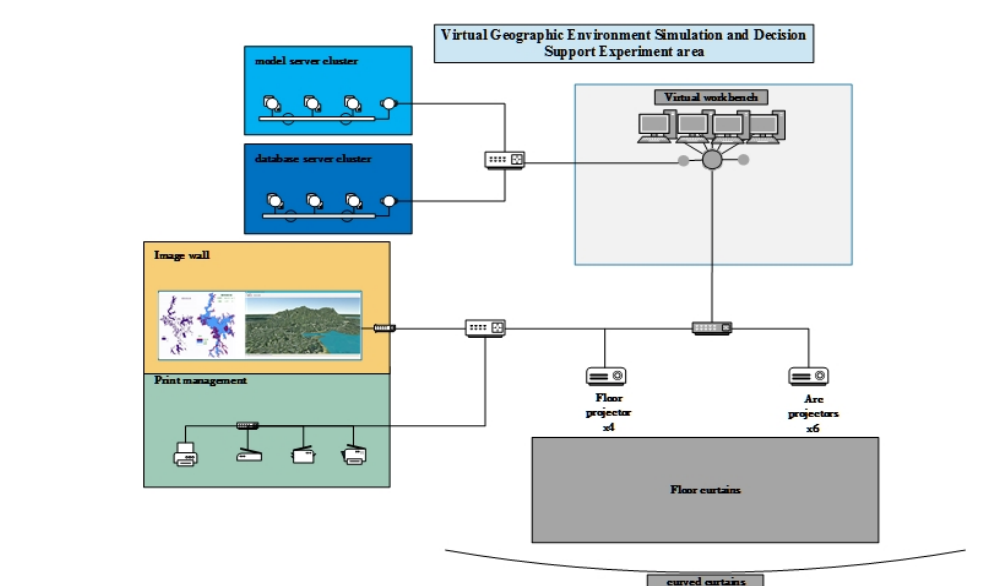

Fig 3 VGE Simulation and Decision Support Experiment area configuration diagram

\section{Conclusions}

Taking the water ecological security of Poyang Lake as the background, taking the VGE as the scientific guide, this paper considers the integration of satellite remote sensing data and model, and provides high-speed calculation and processing for high-performance computing. Based on the IDL language, the water ecological security model is integrated on the computer, and the VGE expression is used to realize the model integration and visualization of the results and output of the results. The long - time scale and spatial scale of the geographical process in the virtual space repeated simulation test, accelerate the maturation and development of geological theory, to achieve the Poyang Lake water ecological security monitoring and early warning.

Features of this paper is to study the Poyang Lake ecological safety ideas VGE first use. Use of virtual geographical environment and safety tests carried out ecological monitoring, not only specific ecological safety, to carry out simulation experiments, to build the real world it is difficult to repeat the incident, complete simulation and forecasting, but also saves the cost of experiments and human and financial resources, which can greatly promote the ecological effectiveness security issues.

\section{Acknowledgements}

This work was financially supported by the Jiangxi Province, Major Ecological Security Issues Coordinated Collaborative Innovation Center (JXS-EW-00), National Surveying and Mapping of Geographic Information Public Welfare Industry Research Special (201512026).

\section{References}

[1] Siquan Yang, Angsheng Wang, Shouting Gao, Yongfeng Liao,Development of Research on the Information System of Disaster Evaluation[J]. Journal of Catastrophology. 2002,17(2):70-75.

[2] Baudrillard J. Simulacra and Simulation[M]. University of Michigan Press, 1994.1-42.

[3] Veen HAHCV, Distler HK, Braun S J, et al. Navigating through a virtual city: Using virtual reality technology to study human action and perception [J]. Future Generation Computer Systems, 1998, 14(3-4):231-242.

[4] Siong Yi, Liangtao Nie: Digital Railway Location System Based on Virtual Geographic Environment (Journal of Southwest Jlaotong University, ChengDu 2016), in Chinese.

[5] Hui Lin, Bingli Xu: Some Thoughts on Virtual Geographic Environments (Geography and Geo-Information Science, HongKong 2007), in Chinese.

[6] Hui Lin, Jianhua Gong: On Virtual Geographic Environments (Acta Geodaetica et Cartographica Sinica, HongKong 2002 ), in Chinese 